\title{
Music Genre Classification Using MIDI and Audio Features
}

\author{
Zehra Cataltepe, Yusuf Yaslan, and Abdullah Sonmez \\ Computer Engineering Department, Faculty of Electrical and Electronic Engineering, Istanbul Technical University, \\ Maslak, Sariyer, Istanbul 34469, Turkey
}

Received 1 December 2005; Revised 17 October 2006; Accepted 19 October 2006

Recommended by George Tzanetakis

We report our findings on using MIDI files and audio features from MIDI, separately and combined together, for MIDI music genre classification. We use McKay and Fujinaga's 3-root and 9-leaf genre data set. In order to compute distances between MIDI pieces, we use normalized compression distance (NCD). NCD uses the compressed length of a string as an approximation to its Kolmogorov complexity and has previously been used for music genre and composer clustering. We convert the MIDI pieces to audio and then use the audio features to train different classifiers. MIDI and audio from MIDI classifiers alone achieve much smaller accuracies than those reported by McKay and Fujinaga who used not NCD but a number of domain-based MIDI features for their classification. Combining MIDI and audio from MIDI classifiers improves accuracy and gets closer to, but still worse, accuracies than McKay and Fujinaga's. The best root genre accuracies achieved using MIDI, audio, and combination of them are $0.75,0.86$, and 0.93 , respectively, compared to 0.98 of McKay and Fujinaga. Successful classifier combination requires diversity of the base classifiers. We achieve diversity through using certain number of seconds of the MIDI file, different sample rates and sizes for the audio file, and different classification algorithms.

Copyright ( 2007 Hindawi Publishing Corporation. All rights reserved.

\section{INTRODUCTION}

The increase of the musical databases on the Internet and multimedia systems have brought a great demand for music information retrieval (MIR) applications and especially automatic analysis of the musical databases. Most of the current databases are indexed based on song title or artist name, where improper indexing can cause incorrect search results. More effective systems extract important features from audio and then based on these features classify the audio to its genre. This kind of music retrieval systems should also have the ability to find similar songs based on their extracted features. However, there are not any strict distinguishing boundaries between audio genres and no complete agreement exists in their definition $[1,2]$.

Generally, music audio signals can be represented in two ways on computers. The first one is symbolic representation based on musical scores. Examples of this representation are MIDI and Humdrum where for each note, pitch, duration (start time/end time), and strength are kept in the file. The second one is based on acoustic signals, recording the audio intensity as a function of time sampled at a certain frequency and can be incompressed or uncompressed format. Because of the difference of the representation of symbolic and acous- tic data, algorithms that deal with data in these formats also differ from each other.

MIDI format developed as a standard to play music on digital instruments or computer. The sound quality of a MIDI music piece depends on the synthesizer (sound card) and MIDI has its other limitations, such as it cannot store voice. On the other hand, this format takes a lot less space, hence it is much easier to store and communicate, is widely accepted, and allows for better comparison between music pieces played on different instruments. Studies on MIDI genre classification date back to the late 1990s [3], see also, for example, $[2,4,5]$.

Recently, [6,7] have suggested using an approximation to Kolmogorov distance between two musical pieces as a mean to compute clusters of music. They first process the MIDI representation of a music piece to turn it into a string from a finite alphabet. Then they compute the distance between two music pieces using their normalized compression distance (NCD). NCD uses the compressed length of a string as an approximation to its Kolmogorov complexity. Although the Kolmogorov complexity of a string is not computable, the compressed length approximation seems to have given good results for a number of data sets ranging from time series to text to video [8]. 
Acoustic music signals are represented using different audio formats, such as VAW, MP3, AAC, or OGG. MP3 compression is the MPEG-1 audio layer 3 compression standard that eliminates the frequencies which are not heard by the human ear. MP3 uses perceptual audio coding and psychoacoustic compression to remove the inaudible parts of the signal [9]. Advanced audio coding (AAC) is the improved codec of the MP3 standard. On the other, hand OGG is a free open-source audio encoding and streaming technology (http://www.vorbis.com). Note that, since MP3, AAC, and OGG are lossy compression methods, the extracted features would be different from the original features. Most of the MIR methods using audio signals have two processing steps. The first one is a frame-based feature extraction step of acoustic data where feature vectors of low-level descriptors are computed from each frame. In the second step, pattern recognition algorithms are applied on the feature vectors to infer the genre. Music genre classification using audio signals has also been widely studied, see, for example, [10-15].

Previously, McKay and Fujinaga [4] have reported very good root (98\%) and leaf (90\%) genre classification accuracy on their 3-root and 9-leaf genre dataset of 225 MIDI music pieces. We use the same data set in our experiments. We first train classifiers for MIDI genre classification. We produce audio files from MIDI files and then use the audio to determine the genres. We combine MIDI and audio classifiers to achieve better accuracy.

We use our preprocessing method $[16,17]$ of MIDI files, compute NCD between them using complearn software (http://www.complearn.org), and then $k$-nearest neighbour classifier to predict root and leaf genre of MIDI files. In order to achieve classifier diversity, we train four different MIDI classifiers, using the first 30 seconds, 60 seconds, 120 seconds of the pieces only and also using the whole piece.

We convert the MIDI files to aiff files using QuickTime Player and Audio Hijack. Then, we use iTunes to obtain wav encoded mono files using 6 different sample rates and sample sizes $(22.050 \mathrm{kHz}, 8$ bit; $22.050 \mathrm{kHz}, 16$ bit; $32 \mathrm{kHz}, 8$ bit; $32 \mathrm{kHz}, 16$ bit; $44.1 \mathrm{kHz}, 8$ bit; $44.1 \mathrm{kHz}, 16$ bit). We use the freely available Marsyas software (http://opihi.cs.uvic.ca/marsyas), by Tzanetakis [12] to extract the audio features.

The rest of the paper is organized as follows: in Section 2, we give brief information on the classifiers we use in our experiments. Section 3 includes the features we used and the classification accuracies we obtain for genre classification of the MIDI-to-audio converted music pieces. In Section 4, we report the results for MIDI genre classification using NCD. Section 5 explains the methods and results for combination of audio and MIDI classifiers. Section 6 concludes the paper.

\section{CLASSIFIERS}

Many classification techniques have been used for genre classification. Examples are: Gaussian mixture models [12], support vector machines [13, 18], radial basis functions [19], linear discriminant analysis [18], and $k$-nearest neighbors [18]. In this study, we report our experiments with linear discriminant classifiers (LDC) which assume normal densities and $k$-nearest neighbor classifiers (KNN). We also have experimented with quadratic discriminant classifiers (QDC), fisher linear discriminant (Fisher), naïve bayes classifier (NBC), and parzen density-based classifier (PDC). However, since they gave as good results and are simpler, in this study, we report our experiments using LDC and KNN. We give brief descriptions of LDC and KNN classifiers below and refer the reader to [20] for more information.

\section{Linear discriminant classifier}

The objective of the linear discriminant analysis is to find sets of hyperplanes separating classes. LDC is a linear classifier assuming normal densities with equal covariance matrices. Fisher's LDA performs dimensionality reduction while preserving the class discriminatory information.

\section{k-nearest neighbor}

Is a well-known nonparametric classifier. The training data is stored with their labels. A new input $x$ is classified according to the labels of its closest (according to a distance metric) $k$ neighbors in the training set. The value of $k$ affects the complexity of the classifier. In our experiments, we use $k=10$ $(10 \mathrm{NN})$.

\section{GENRE CLASSIFICATION USING AUDIO FEATURES}

Several feature extraction methods including low-level parameters such as zero-crossing rate, signal bandwidth, spectral centroid, root mean-square level, band energy ratio, delta spectrum, psychoacoustic features, MFCC, and auditory filterbank temporal envelopes have been employed for audio classification [12]. Today's state-of-the-art audio genre classification methods are evaluated at music information retrieval evaluation exchange (MIREX) contests, see, for example, [21]. In our experiments, we have obtained the following content-based audio features using Tzanetakis's Marsyas software.

\subsection{Timbral features}

Timbral features are generally used for music-speech discrimination and speech recognition. They differentiate mixture of sounds with the same or similar rhythmic content. In order to extract the timbral features, audio signal is divided into small intervals that can be acceptable as stationary signal. The following timbral features are calculated for these small intervals.

(i) Spectral centroid: measures the spectral brightness and is defined as the center of the gravity of the magnitude spectrum of the STFT.

(ii) Spectral rolloff: measures the spectral shape and is defined as the frequency value below which lies the $85 \%$ of the magnitude distribution.

(iii) Spectral flux: measures the amount of local spectral change and is defined as the squared difference between the normalized magnitudes of successive spectral distributions. 
(iv) Time domain zero crossing: measures the noisiness of the signal and is defined as the number of time domain zero crossings of the signal.

(v) Low energy: measures the amplitude distribution of the signal and is defined as the percentage of the frames that have RMS energy less than the average RMS energy over the whole signal.

(vi) Mel-frequency cepstral coefficients (MFCC): MFCCs are well known for speech representation. They are calculated by taking the log-amplitude of the magnitude spectrum and then smoothing the grouped FFT bins according to the perceptually motivated Mel-frequency scaling.

Means and variances of the spectral centroid, spectral rolloff, spectral flux, zero crossing (8 features), and low energy (1 feature) results in 9-dimensional feature vector and represented in experimental results as STFT label [12]. Means and variances of the first five MFCC coefficients yield a 10-dimensional feature vector, which is represented as MFCC in the experiments.

\subsection{Rhythmic content features}

Rhythmic content features characterize the movement of music signals over time and contain such information as the regularity of the rhythm, the beat, the tempo, and the time signature $[12,22]$. The feature set for representing rhythm structure is based on detecting the most salient periodicities of the signal. Rhythmic content features are calculated by beat histogram calculation and yield a 6-dimensional feature vector which is represented using BEAT label.

\subsection{Pitch content features}

The melody and harmony information about the music signal is obtained by pitch detection techniques. Although musical genres by no means can be characterized fully by their pitch content, there are certain tendencies that can lead to useful feature vectors [12]. Pitch content features are calculated by pitch histogram calculation and yield a 5dimensional feature vector which is represented as MPITCH in the experimental results.

The following is a list of audio features we use and their length:

(i) BEAT ( 6 features),

(ii) STFT (9 features),

(iii) MFCC (10 features),

(iv) MPITCH (5 features),

(v) ALL (30 features).

\subsection{Effect of sample rate and size on genre classification}

When an audio file is compressed under different settings, its features could change. In order to understand what changes could happen, we used different sample rates $(22.050 \mathrm{kHz}, 32 \mathrm{kHz}, 44.1 \mathrm{kHz})$, sample sizes ( 8 bit, 16 bit) to convert the audio file to wav format. As seen in Figure 1, we examined the normalized mean difference between features on all data points using one setting versus another setting.

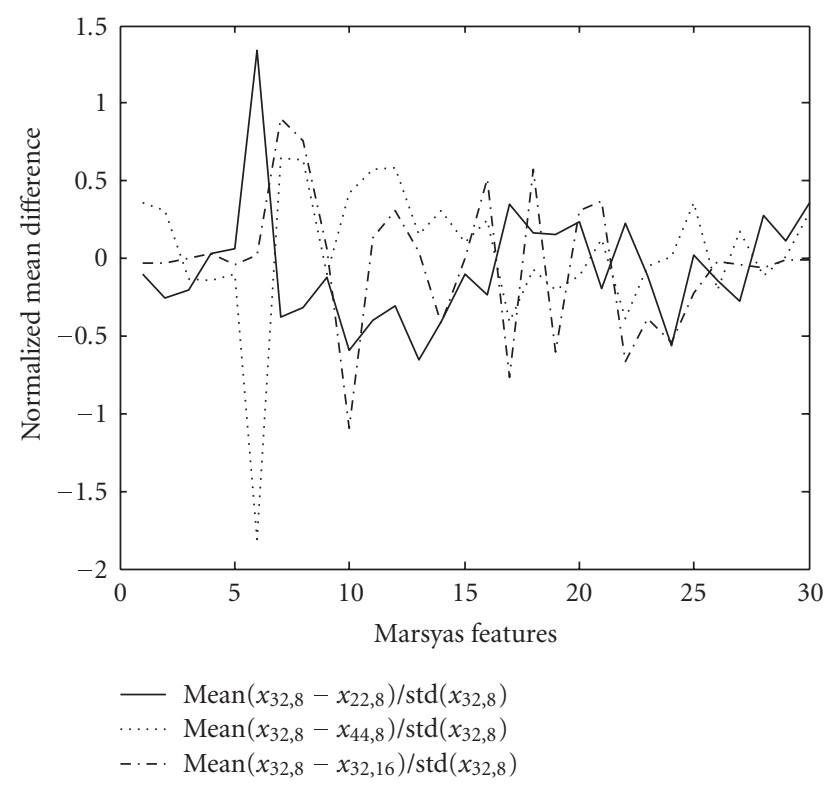

Figure 1: The change of Marsyas features when different sample rates and sample sizes are used.

There is some variability on all the features, although features 6 (BEAT), 7, 8 and 10 (STFT) seem to vary more than others.

In order to understand the effect of feature changes due to compression settings, we trained different classifiers using different feature sets (ALL, BEAT, MFCC, MPITCH, STFT) obtained under different compression settings. In Figures 2 and 3 , the $x$-axis shows different audio sampling rates and sizes: 1 : $22.05 \mathrm{kHz}, 8$ bit; $2: 22.05 \mathrm{kHz}, 16$ bit; 3 : $32 \mathrm{kHz}$, 8 bit; $4: 32 \mathrm{kHz}, 16$ bit; $5: 44.1 \mathrm{kHz}, 8$ bit, $6: 44.1 \mathrm{kHz}, 16$ bit. For each genre, $90 \%$ of all available data was used for training and $10 \%$ was used for testing. In the figures and tables below, the test classification accuracies are reported. Using ALL features almost always gave better performance than using one of the other specified feature sets. MFCC's performance was very close to that of ALL, though. MPITCH and BEAT usually gave the least classification accuracy. When ALL features were used, we found out that the expected performance did not change a lot between different sample rates and sizes.

Table 1 shows the root and leaf genre classification accuracies obtained using the first and last two $(22.05 \mathrm{kHz}$ or $44.1 \mathrm{kHz}$ and 8 or 16 bits) compression settings. LDC performs better than $10 \mathrm{NN}$ for both root and genre classification.

\section{GENRE CLASSIFICATION USING MIDI AND NCD}

One way to measure the distance between two music pieces is to first extract features and then measure distance between feature vectors. For example, [4] uses 109 features of musical information such as orchestration, number of instruments, adjacent fifths, and so forth. Once distances are available, a classification algorithm, such as $k$-nearest neighbor, can be used to predict the genre of a music piece. 


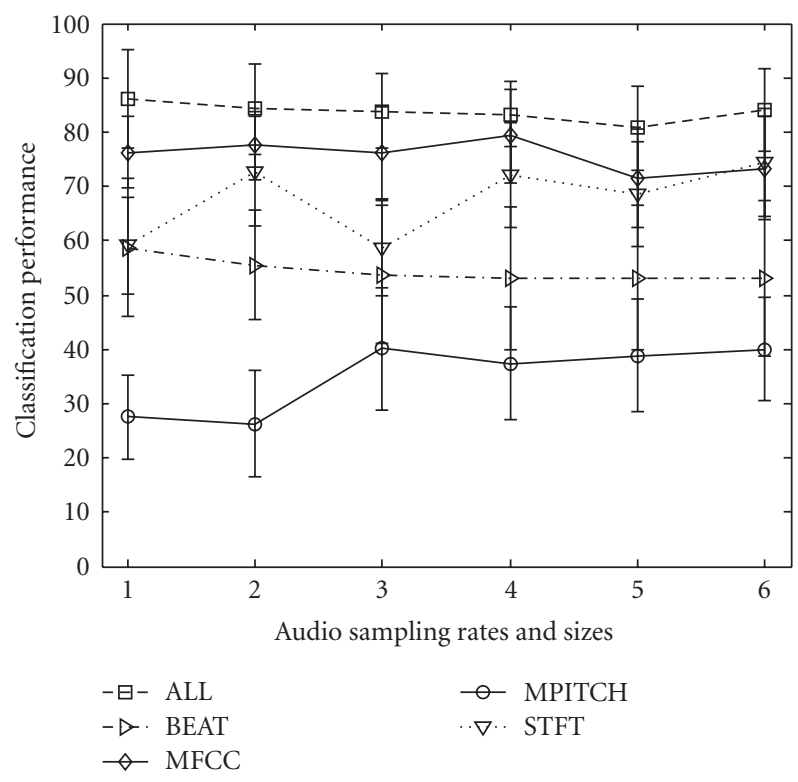

FIgURE 2: Root genre test classification accuracies of LDC classifier using different sets of features (each curve) at different audio sampling rates and sizes ( $x$-axis).

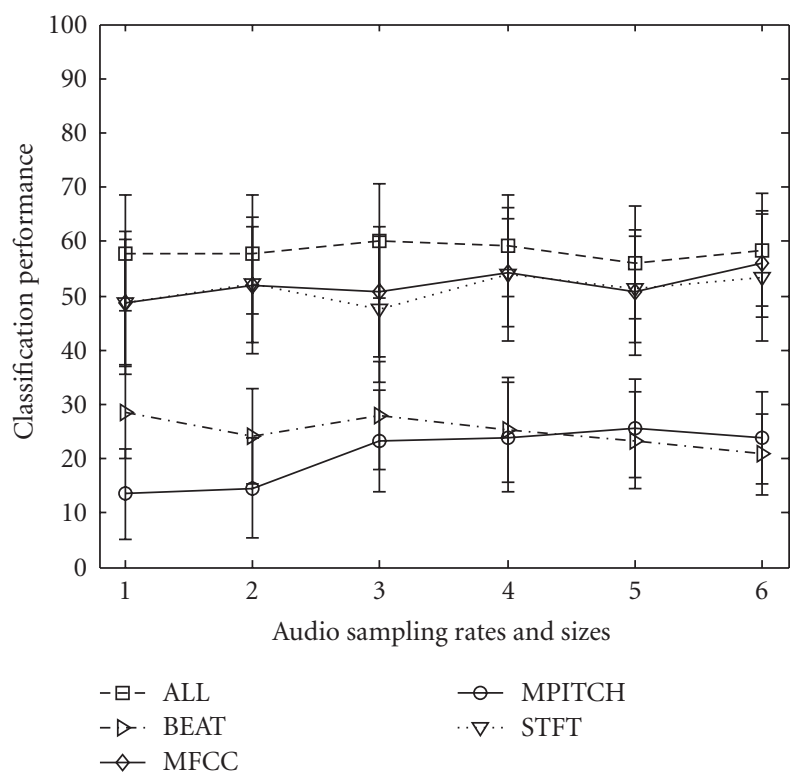

FIgURE 3: Leaf genre test classification accuracies of LDC classifier using different sets of features (each curve) at different audio sampling rates and sizes ( $x$-axis).

In this study, in order to measure the distance between two music pieces, we use normalized compression distance (NCD). According to NCD, two objects are said to be close if the information contained in one of them can be compressed in the other. In other words, if two pieces are similar, then it is possible to describe one given the other. The compression is based on the ideal mathematical notion of Kolmogorov complexity, which unfortunately is not effectively computable.
TABLE 1: Root and leaf genre test classification accuracies on audio data obtained from MIDI, using different compression settings and $10 \mathrm{NN}$ and LDC classifiers.

\begin{tabular}{l|llll}
\hline Audio & $\begin{array}{l}22.05 \mathrm{kHz}, \\
8 \text { bits (1) }\end{array}$ & $\begin{array}{l}22.05 \mathrm{kHz}, \\
16 \text { bits (2) }\end{array}$ & $\begin{array}{l}44 \mathrm{kHz}, \\
8 \text { bits(5) }\end{array}$ & $\begin{array}{l}44 \mathrm{kHz}, \\
16 \operatorname{bits}(6)\end{array}$ \\
\hline Root, 10 NN & $0.52 \pm 0.01$ & $0.53 \pm 0.01$ & $0.54 \pm 0.01$ & $0.58 \pm 0.01$ \\
Root, LDC & $0.86 \pm 0.01$ & $0.84 \pm 0.01$ & $0.83 \pm 0.01$ & $0.86 \pm 0.01$ \\
Leaf, 10 NN & $0.19 \pm 0.01$ & $0.20 \pm 0.01$ & $0.23 \pm 0.01$ & $0.30 \pm 0.01$ \\
Leaf, LDC & $0.59 \pm 0.01$ & $0.63 \pm 0.01$ & $0.60 \pm 0.01$ & $0.63 \pm 0.01$ \\
\hline
\end{tabular}

TABLE 2: Root and leaf genre test classification accuracies on MIDI data using $10 \mathrm{NN}$ classifier with NCD.

\begin{tabular}{l|cccc}
\hline MIDI & 30 seconds & 60 seconds & 120 seconds & ALL \\
\hline Root & $0.67 \pm 0.01$ & $0.66 \pm 0.01$ & $0.67 \pm 0.01$ & $0.75 \pm 0.01$ \\
Leaf & $0.31 \pm 0.01$ & $0.39 \pm 0.01$ & $0.46 \pm 0.01$ & $0.42 \pm 0.01$ \\
\hline
\end{tabular}

However, it is possible to approximate the Kolmogorov complexity by using standard compression techniques. NCD uses no background knowledge about music, it is completely general and can, without change, be used in different areas like linguistic classification and genomics.

In $[6,7]$, first the MIDI representation of a music piece is processed and transformed into a string from a finite alphabet. Then the distance between two music pieces $x$ and $y$ are computed using their NCD:

$$
d(x, y)=\frac{\max \{K(x \mid y), K(y \mid x)\}}{\max \{K(x), K(y)\}} .
$$

In this formula, $K(x)$ denotes the Kolmogorov complexity of $x$ and $K(x \mid y)$ denotes the Kolmogorov complexity of $x$ given $y . K(x \mid y)$ is approximated using $K(x \mid y) \approx K(x y)-$ $K(x)$. NCD uses the compressed length of a string as an approximation of its Kolmogorov Complexity. $K(x y)$ is computed simply as the compressed length of $x$ and $y$ concatenated together. This compressed length approximation to Kolmogorov complexity seems to have given good results for a number of different data sets in [8].

In this study, we use our preprocessor $[16,17]$ on MIDI files to turn them into strings. The MIDI preprocessor samples the MIDI file at each $5 \mathrm{~ms}$. and discovers the notes simultaneously played at each interval. It converts each note played in that interval to an integer between 0 and 127. Since all pieces used in experiments are polyphonic, like in most of the cases in the real world, polyphonic to monophonic conversion is needed. The note which is heard as the highest pitch [23] is taken as the representative of the interval. Then the difference between consecutive monophonic notes is taken and written to a binary file. Apart from [6, 7], tempo variations are taken into account and difference between consecutive monophonic notes is taken. Like them, we use NCD as the distance measure between two pieces.

Table 2 shows the root and leaf genre classification accuracy of the $10 \mathrm{NN}$ classifier using NCD as the distance 


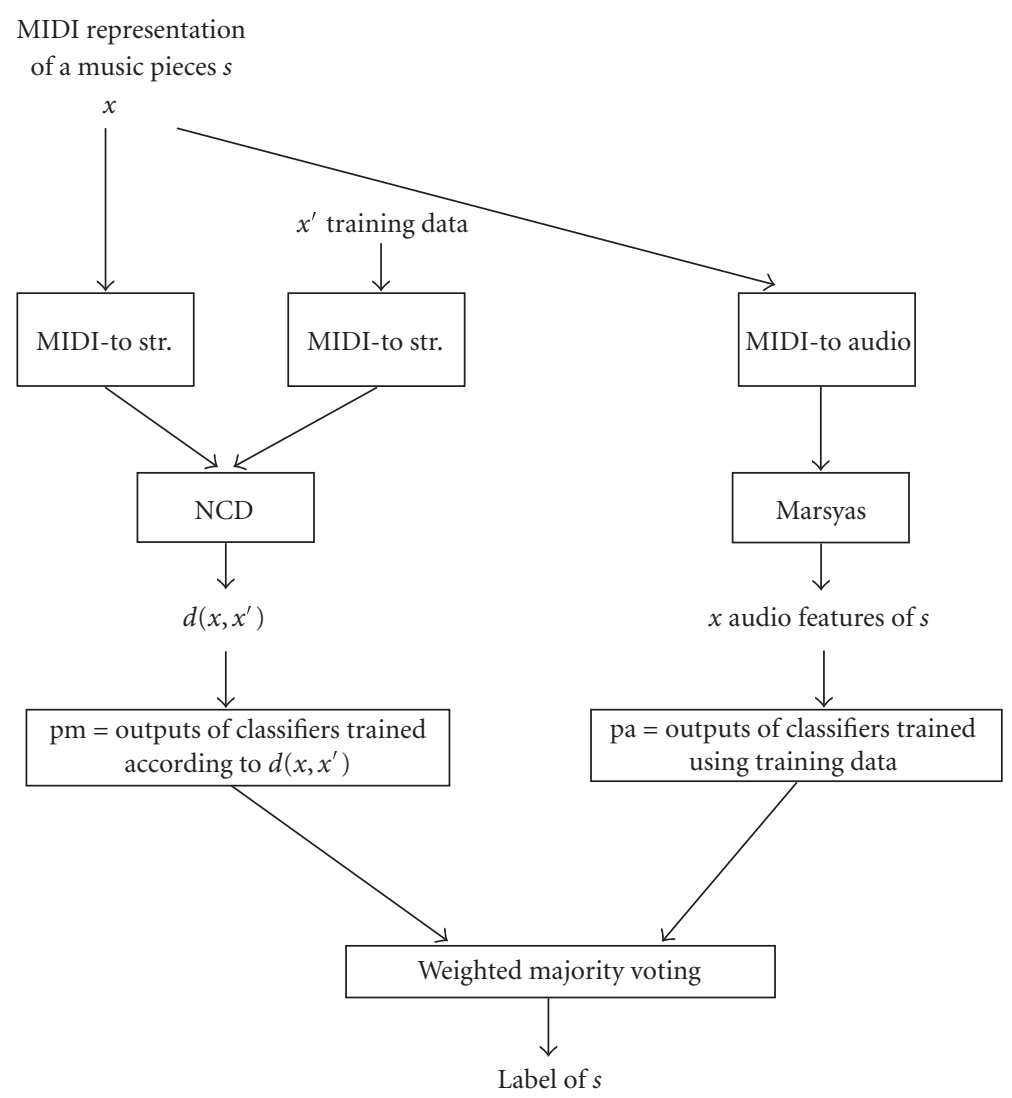

FIGURE 4: A method to combine MIDI and audio features to predict the genre of a MIDI music piece.

measure. Distances are computed using the first 30, 60, 120 seconds and finally using all the available music piece. The accuracies shown are computed over 100 different train/test partitions of all the available data. Using the whole piece results in the best root genre classification performance, while using only the first 120 seconds results in the best leaf genre classification performance. Note that, as in the case of the previous section, the root and leaf genre classification performances are quite below the results obtained in [4].

\section{GENRE CLASSIFICATION USING BOTH MIDI AND AUDIO FROM MIDI}

We explored the root and leaf genre classification accuracy using MIDI and audio separately and found out that the accuracy varied between different feature sets and classifiers. However, the accuracies reached were far below the accuracies obtained in [4]. In this section, we investigate if we can get better results by combining MIDI and audio classifiers we obtained in the previous two sections.

According to Kuncheva [24], in order for classifier combination to be successful, classifiers need to be diverse. The probability that many classifiers, trained independently, will agree on the same wrong output is small. Therefore, majority voting could give the right answer for the many, independent and diverse classifiers case.
There are a number of methods to achieve diverse classifiers: (a) use independent sub samples of data to train each classifier, (b) use different sets of features to train each classifier, (c) use different algorithms to train each classifier. In this paper, we use (b) and (c) to achieve classifier diversity.

MIDI distances and audio features give us an initial base of different features. We get still more different features by using different initial portions of the MIDI file and different sample rates and sizes for the audio file. The $k$-nearest neighbor and LDC classifiers also help achieve more diversity. Therefore, we have a pool of different classifiers whose votes we can combine to achieve better accuracy (Figure 4).

Let $D_{i}, i=1, \ldots, L$, indicate the different trained classifiers. In this paper, $L=12$ and $i=1: 4$ correspond to $10 \mathrm{NN}$ classifiers, trained using NCD between MIDI files. $i=5: 8$ corresponds to $10 \mathrm{NN}$ classifiers, trained using all 30 features. $i=9: 12$ corresponds to linear discriminant classifiers trained, again, using all 30 features. Let $d_{i, j}$ be 1 if classifier $i$ labels $x$ in class $j$ and 0 otherwise. Let $w_{i}$ denote the weight of classifier $i$. The weighted majority voting chooses class $j^{*}$ such that

$$
j^{*}=\arg \max _{j=1, \ldots, L} \sum_{i=1, \ldots, C} w_{i} d_{i, j} .
$$

We consider four different flavors of weighted majority voting described by the weights $w_{i}$ given to each classifier. 
TABLE 3: Root and leaf genre classification accuracies when classifiers are combined.

\begin{tabular}{l|cccc}
\hline $\begin{array}{l}\text { MIDI } \\
\text { and }\end{array}$ & $w_{i}=1$ & $\begin{array}{c}i=1-4: w_{i}=2 \\
i=5-8: w_{i}=1 \\
i=9-12: w_{i}=2\end{array}$ & $w_{i} \alpha$ acc $_{i}$ & $w_{i}$ optimal \\
audio & & $0.89 \pm 0.01$ & $0.89 \pm 0.01$ & $0.93 \pm 0.01$ \\
\hline Root & $0.88 \pm 0.01$ & $0.58 \pm 0.01$ & $0.58 \pm 0.01$ & $0.62 \pm 0.01$ \\
Leaf & $0.58 \pm 0.01$ & &
\end{tabular}

TABLE 4: Root genre confusion matrices for 12 different base classifiers.

\begin{tabular}{|c|c|c|c|c|c|c|c|c|c|c|}
\hline \multirow{2}{*}{ No } & \multirow{2}{*}{ Feature, classifier } & \multicolumn{3}{|c|}{ Actual $=$ classic } & \multicolumn{3}{|c|}{ Actual $=\mathrm{jazz}$} & \multicolumn{3}{|c|}{ Actual $=$ pop } \\
\hline & & Pred class & Pred jazz & Pred pop & Pred class & Pred jazz & Pred pop & Pred class & Pred jazz & Pred pop \\
\hline 1 & MIDI, $30 \mathrm{~s}, 10 \mathrm{NN}$ & 89 & 7 & 4 & 14 & 82 & 4 & 45 & 26 & 29 \\
\hline 2 & MIDI, $60 \mathrm{~s}, 10 \mathrm{NN}$ & 69 & 13 & 18 & 6 & 86 & 8 & 25 & 33 & 42 \\
\hline 3 & MIDI, $120 \mathrm{~s}, 10 \mathrm{NN}$ & 70 & 4 & 26 & 8 & 76 & 16 & 20 & 23 & 56 \\
\hline 4 & MIDI, ALL, $10 \mathrm{NN}$ & 75 & 4 & 21 & 6 & 84 & 10 & 13 & 22 & 66 \\
\hline 5 & Audio, $22,8,10 \mathrm{NN}$ & 72 & 13 & 15 & 10 & 48 & 41 & 21 & 42 & 37 \\
\hline 6 & Audio, $22,16,10 \mathrm{NN}$ & 71 & 6 & 23 & 12 & 41 & 47 & 19 & 34 & 47 \\
\hline 7 & Audio, $44,8,10 \mathrm{NN}$ & 63 & 15 & 22 & 8 & 53 & 39 & 14 & 39 & 47 \\
\hline 8 & Audio, $44,16,10 \mathrm{NN}$ & 69 & 12 & 19 & 14 & 46 & 40 & 10 & 30 & 60 \\
\hline 9 & Audio, 22, 8, LDC & 94 & 3 & 2 & 6 & 88 & 6 & 12 & 13 & 75 \\
\hline 10 & Audio, 22, 16, LDC & 96 & 0 & 4 & 3 & 87 & 10 & 12 & 20 & 68 \\
\hline 11 & Audio, 44, 8, LDC & 98 & 1 & 2 & 2 & 82 & 16 & 9 & 21 & 70 \\
\hline 12 & Audio, 44, 16, LDC & 97 & 0 & 3 & 4 & 82 & 14 & 4 & 18 & 78 \\
\hline
\end{tabular}

(i) $w_{i}=1$ : this voting scheme gives each classifier the same amount of vote.

(ii) $w_{i}=2$ if $1 \leq i \leq 4$ or $9 \leq i \leq 12$ and $w_{i}=1$ if $5 \leq i \leq 8$ : inspired by the fact that audio- $10 \mathrm{NN}$ gives the worst results, this method gives less weight to those classifiers.

(iii) $w_{i}$ proportial to accuracy of $i$ th classifier: this method depends on the accuracy of each classifier which is not available. However, using a subset of training data for validation accuracy could be estimated.

(iv) $w_{i}$ selected to maximize accuracy: this method exhaustively searches the $w_{i}$ 's in $[0.2: 1]$ interval and reports the $w_{i}$ that results in the best accuracy. This method is also not realizable in practice, however, it is included to report the best possible performance using weighted majority voting.

Table 3 shows the leaf and root genre classification accuracies of each classifier combination method. Comparison of Tables 1,2 , and 3 shows that root genre classification accuracy increases when classes are combined for all of the combination schemes.

Table 4 shows the confusion matrix entries for each of the base classifiers. The entries are averaged over 100 train/test partitions and normalized to 100 per actual class. Each row corresponds to a classifier with a different feature and classification method. Second column shows whether the MIDI or audio input is used and the type of classifier used. This column also shows the length of the used piece for MIDI and the sample rate and sample size for audio. Although the accuracies were similar, clearly the confusion matrices are dif- ferent for each feature-classifier combination and this helped combination achieve better results. Another observation is that classic is recognized best when 30 seconds of MIDI file is used, whereas pop benefits from longer files. While higher quality (i.e., more $\mathrm{kHz}$ and 16 bits) encoding usually helps classic and pop, the same is not true for jazz.

Table 5 shows the confusion matrices for the classifier combinations. Using audio and LDC usually gave the best results on Table 4, and Table 5's entries are better than that. Choosing classifier weights according to accuracies did not improve over the equal-weighted majority voting. On the other hand, choosing the optimal weights according the specific set of samples being classified resulted in better performance.

\section{CONCLUSIONS}

In this paper, we first classified genres using MIDI files using normalized compression distance (NCD) and 10-nearest neighbor $(10 \mathrm{NN})$ classifier. We converted MIDI files to audio and did genre classification using features at different sample rates and sizes and LDC and KNN classifiers. Finally, we combined 12 different classifiers we obtained at the previous steps, using different schemes of majority voting. We found out that majority voting improved the classification accuracy. The classification accuracies for MIDI or audio only were much below the results obtained in [4]. Classifier combination improved genre classification, although the results are still worse than those reported by [4] on their data sets. Since 109 different domain-based features such as orchestration, number of instruments, adjacent fifths, and so 
TABLE 5: Root genre confusion matrices for four different combinations of base classifiers.

\begin{tabular}{|c|c|c|c|c|c|c|c|c|c|}
\hline \multirow[b]{2}{*}{ Combination method } & \multicolumn{3}{|c|}{ Actual $=$ classic } & \multicolumn{3}{|c|}{ Actual $=$ jazz } & \multicolumn{3}{|c|}{ Actual $=$ pop } \\
\hline & Pred class & Pred jazz & Pred pop & Pred class & Pred jazz & Pred pop & Pred class & Pred jazz & Pred pop \\
\hline $\begin{array}{l}w_{i}=1 \\
i=1-4: w_{i}=2\end{array}$ & 99 & 0 & 1 & 3 & 93 & 4 & 8 & 19 & 72 \\
\hline $\begin{array}{l}i=5-8: w_{i}=1 \\
i=9-12: w_{i}=2\end{array}$ & 99 & 0 & 1 & 3 & 93 & 4 & 8 & 17 & 76 \\
\hline$w_{i} \alpha \operatorname{acc}_{i}$ & 99 & 0 & 1 & 3 & 93 & 4 & 8 & 17 & 76 \\
\hline$w_{i}$ optimal & 100 & 0 & 0 & 2 & 94 & 3 & 5 & 10 & 86 \\
\hline
\end{tabular}

forth were used in [4], and, for example, instrumentation features were assigned up to $42 \%$ weight among their features, we think that our results could be improved if instead of using NCD, we used features similar to those reported in [4]. We should also note that, in contrast to [4], the approach outlined in this paper does not require any musical background knowledge.

Currently, the audio to MIDI conversion is not very successful, especially when multiple instruments are used in the piece. We hope that as technology gets better, a similar approach that combines audio and audio-to-MIDI features could be used to improve audio genre classification.

\section{ACKNOWLEDGMENTS}

We would like to express our gratitude to George Tzanetakis and Cory McKay for generously sharing their data sets. We also would like to thank Tzanetakis for Marsyas, Cilibrasi, and colleagues for Complearn and Bob Duin and colleagues for PrTools, which was used in some of the experiments. We thank the reviewers for helping us improve the quality of the paper.

\section{REFERENCES}

[1] S. Lippens, J. P. Martens, and T. De Mulder, "A comparison of human and automatic musical genre classification," in Proceedings of IEEE International Conference on Acoustics, Speech and Signal Processing (ICASSP '04), vol. 4, pp. 233-236, Montreal, Quebec, Canada, May 2004.

[2] R. Basili, A. Serafini, and A. Stellato, "Classification of musical genre: a machine learning approach," in Proceedings of the 5th International Conference on Music Information Retrieval (ISMIR '04), Barcelona, Spain, October 2004.

[3] T. Jarvinen, P. Toiviainen, and J. Louhivuori, "Classification and categorization of musical styles with statistical analysis and self-organizing maps," in Proceedings of the AISB Symposium on Musical Creativity, pp. 54-57, Edinburgh, Scotland, April 1999.

[4] C. McKay and I. Fujinaga, "Automatic genre classification using large high-level musical feature sets," in Proceedings of 5th International Conference on Music Information Retrieval (ISMIR '04), Barcelona, Spain, October 2004.

[5] G. Tzanetakis, A. Ermolinskyi, and P. Cook, "Pitch histograms in audio and symbolic music information retrieval," Journal of New Music Research, vol. 32, no. 2, pp. 143-152, 2003.
[6] R. Cilibrasi, P. M. B. Vitányi, and R. de Wolf, "Algorithmic clustering of music based on string compression," Computer Music Journal, vol. 28, no. 4, pp. 49-67, 2004.

[7] M. Li, X. Chen, X. Li, B. Ma, and P. M. B. Vitányi, "The similarity metric," IEEE Transactions on Information Theory, vol. 50, no. 12, pp. 3250-3264, 2004.

[8] E. Keogh, S. Lonardi, and C. A. Rtanamahatana, "Towards parameter-free data mining," in Proceedings of the 10th ACM SIGKDD International Conference on Knowledge Discovery and Data Mining (KDD '04), pp. 206-215, Seattle, Wash, USA, August 2004.

[9] D. Pan, "A tutorial on MPEG/audio compression," IEEE Multimedia, vol. 2, no. 2, pp. 60-74, 1995.

[10] J. J. Aucouturier and F. Pachet, "Representing musical genre: a state of the art," Journal of New Music Research, vol. 32, no. 1, pp. 83-93, 2003.

[11] T. Lidy and A. Rauber, "Evaluation of feature extractors and psycho-acoustic transformations for music genre classification," in Proceedings of the 6th International Conference on $\mathrm{Mu}$ sic Information Retrieval (ISMIR '05), London, UK, September 2005.

[12] G. Tzanetakis and P. Cook, "Musical genre classification of audio signals," IEEE Transactions on Speech and Audio Processing, vol. 10, no. 5, pp. 293-302, 2002.

[13] C. Xu, N. C. Maddage, X. Shao, F. Cao, and Q. Tian, "Musical genre classification using support vector machines," in Proceedings of IEEE International Conference on Acoustics, Speech and Signal Processing (ICASSP '03), vol. 5, pp. 429-432, Hong Kong, April 2003.

[14] F. Gouyon, S. Dixon, E. Pampalk, and G. Widmer, "Evaluating rhythmic descriptors for musical genre classification," in Proceedings of the 25th International AES Conference, London, UK, June 2004.

[15] K. West and S. Cox, "Features and classifiers for the automatic classification of musical audio signals," in Proceedings of the 5th International Conference on Music Information Retrieval (ISMIR '04), Barcelona, Spain, October 2004.

[16] A. Sonmez, "Music genre and composer identification by using Kolmogorov distance," M. Sc. thesis, Computer Engineering Department, Istanbul Technical University, Istanbul, Turkey, 2005.

[17] Z. Cataltepe, A. Sonmez, and E. Adali, "Music classification using Kolmogorov distance," in Representation in Music/Musical Representation Congress, Istanbul, Turkey, October 2005.

[18] T. Li, M. Ogihara, and Q. Li, "A comparative study on contentbased music genre classification," in Proceedings of the 26th Annual International ACM SIGIR Conference on Research and Development in Information Retrieval (SIGIR '03), pp. 282-289, Toronto, Ontario, Canada, July-August 2003. 
[19] D. Turnbull and C. Elkan, "Fast recognition of musical genres using RBF networks," IEEE Transactions on Knowledge and Data Engineering, vol. 17, no. 4, pp. 580-584, 2005.

[20] R. O. Duda, P. E. Hart, and D. G. Stork, Pattern Classification, John Wiley \& Sons, New York, NY, USA, 2000.

[21] J. Bergstra, N. Casagrande, and D. Eck, "Genre classification: timbre and rhythm-based multiresolution audio classification," in Proceedings of 1st Annual Music Information Retrieval Evaluation eXchange (MIREX) Genre Classification Contest, London, UK, September 2005.

[22] T. Li and G. Tzanetakis, "Factors in automatic musical genre classification of audio signals," in Proceedings of IEEE Workshop on Applications of Signal Processing to Audio and Acoustics (WASPAA '03), New Paltz, NY, USA, October 2003.

[23] L. Uitdenbogerd and J. Zobel, "Music ranking techniques evaluated," Australian Computer Science Communications, vol. 24, no. 1, pp. 275-283, 2002.

[24] L. I. Kuncheva, Combining Pattern Classifiers, John Wiley \& Sons, New York, NY, USA, 2004.

Zehra Cataltepe is an Assistant Professor at Computer Engineering Department, Istanbul Technical University. Her research interests are machine learning theory and applications, especially in bioinformatics, web/ document mining, and music recognition and recommendation. She got her Ph.D. degree from Caltech in computer science in 1998 and her B.S. degree from Bilk-ent University, Ankara, in 1992. She worked at Bell

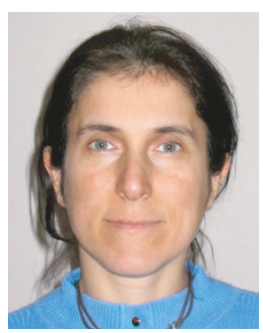
Labs as a postdoc and then at StreamCenter Inc. and Siemens Corporate Research as researcher after she got her Ph.D.

Yusuf Yaslan received the B.S. degree in computer science engineering from Istanbul University, Turkey, in 2001. During 2001 and 2002, he was a practical trainer at the FGAN-FOM Research Institute, in Germany. In 2002, he joined the Multimedia Signal Processing and Pattern Recognition laboratory at Istanbul Technical University (ITU). He received his M.S. degree in telecommunication engineering from ITU,

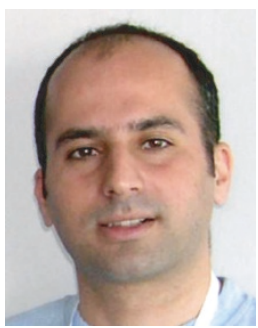
Turkey, in 2004. He is currently working at Computer Engineering Department at ITU as a research assistant, and pursuing his Ph.D. in the same department. His research interests are in pattern recognition, data and web mining, audio watermarking, and music recommendation.

Abdullah Sonmez is a Ph.D. candidate at the Department of Computer Engineering at Istanbul Technical University and currently working in $\mathrm{R} \& \mathrm{D}$ center of Teknobil Inc. as a researcher and developer. His research interests include information retrival especially in music, data mining and machine learning, especially in bioinformatics, GSM and satellite-based communication networks and VoIP. He got his M.S. de-

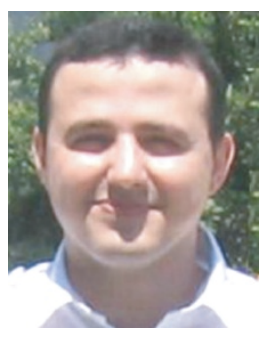
gree from Istanbul Technical University in computer engineering in 2005 and his B.S. degree from Istanbul Technical University in 2002. 